\title{
CRB-65: Predictor for Intensive Care Unit Admission in Patients with Biliary Tract Infection Presenting to An Emergency Department
}

\author{
Hansol Yeo ${ }^{1}$, Sung Jin Bae ${ }^{1}$, Yoon Hee Choi ${ }^{1}$, Keon Kim ${ }^{1}$, Jae Hee Lee ${ }^{1 *}$
}

${ }^{1}$ Department of Emergency Medicine, College of Medicine, Ewha Womans University, Seoul, Republic of Korea

\section{*Correspondence \\ jaeheelee.md@hanmail.net}

(Jae Hee Lee)

\begin{abstract}
Objectives: Biliary tract infection (BTI) is a common cause of bacteremia and is associated with high morbidity and mortality. However, studies on screening tools to predict disease severity in BTI patients are lacking. This study aimed to comparatively validate CRB, CRB-65, quick Sequential Organ Failure Assessment (qSOFA), and Systemic Inflammatory Response Syndrome (SIRS) in predicting the clinical outcomes of BTI patients. Methods: This retrospective cohort study included patients with BTI who visited the emergency department of a medical center between February 2018 and March 2020. Baseline patient data were compared to assess the prevalence of intensive care unit (ICU) admission and in-hospital mortality. The effectiveness of CRB, CRB-65, qSOFA, and SIRS scores as indicators of ICU admission and in-hospital mortality was evaluated using the area under the receiver operating characteristic (AUROC) curve. Results: This study included 745 patients, of whom 111 (14.8\%) were admitted to the ICU and $20(2.7 \%)$ died in-hospital. AUROC values $(95 \% \mathrm{CI})$ for predicting ICU admission and in-hospital mortality were as follows: CRB, 0.774 and 0.707 (0.742 0.803 and $0.673-0.739)$; CRB - 65, 0.816 and 0.735 (0.786- 0.843 and 0.0.702 - 0.766); qSOFA, 0.779 and 0.724 (03747 - 0.808 and $0.690-0.755)$; and SIRS, 0.686 and 0.659 (0.651 - 0.719 and $0.623-0.693)$, respectively. Conclusions: CRB-65 can be used as useful screening tools to predict ICU admission in patients with BTI on presentation to the emergency department.
\end{abstract}

\section{Keywords}

Biliary tract infection, Emergency department, CRB-65, CRB, Quick Sequential Organ Failure Assessment

\section{Introduction}

Sepsis is a common cause for hospitalization and accounts for 750,000 cases annually in America, with the majority of patients presenting through the emergency department (ED) [1]. Among these patients, biliary tract infection (BTI) is the second most frequent cause of sepsis in elderly patients, whereas urinary tract infection is the leading cause [2]. BTI, including cholecystitis and cholangitis, is a common cause of bacteremia and is associated with high morbidity and mortality, particularly in older patients with comorbidities or when there is a delay in diagnosis and treatment [2]. Because BTI is a major cause of sepsis, especially in elderly patients, early screening for disease severity is important [2]. In patients with cholangitis and bacteremia, acute renal failure, septic shock, malignant obstruction, hyperbilirubinemia, and a high Charlson Comorbidity Index score $>6$ were reported to be predictors for mortality [3]. However, as these factors are based on laboratory or radiologic findings, it is difficult to determine the severity at an early stage. In addition, in the case of the Charslon Co-morbidity score, there is a limitation that the score cannot be ascertained in the absence of information on the medical history.

To easily determine the disease severity in the early stages of ED treatment, a scoring system consisting of physiologic parameters without including laboratory finding would be useful. The quick Sequential Organ Failure Assessment (qSOFA) is a well-known scoring system used for the definition of sepsis [4]. The CRB (confusion/respiration rate/blood pressure) and CRB-65 (confusion/respiration rate/blood pressure/65 years older) were developed for community-acquired pneumonia and are tools that have been validated in many studies [5], and the score consist of the patient's mental status, respiration, blood pressure, and age. In this study, we aimed to validate the clinical utility of the CRB, CRB-65, qSOFA, and Systemic Inflammatory Response Syndrome (SIRS) for predicting the clinical outcomes of BTI patients and compare the performance of these screening tests with each other. 
TA B L E 1. General characteristics of study participants.

\begin{tabular}{|c|c|c|c|c|c|c|c|}
\hline \multirow[t]{2}{*}{ Variable } & \multirow{2}{*}{$\begin{array}{c}\text { Total } \\
(\mathrm{n}=745)\end{array}$} & \multicolumn{2}{|c|}{ ICU admission } & \multirow[t]{2}{*}{ p-value } & \multicolumn{2}{|c|}{ In-hospital mortality } & \multirow[t]{2}{*}{ p-value } \\
\hline & & $\begin{array}{l}\text { Non-ICU admission } \\
\qquad(\mathrm{n}=634)\end{array}$ & $\begin{array}{l}\text { ICU admission } \\
\quad(\mathrm{n}=111)\end{array}$ & & $\begin{array}{l}\text { Survivors } \\
(\mathrm{n}=725)\end{array}$ & $\begin{array}{l}\text { Non-survivors } \\
\quad(\mathrm{n}=20)\end{array}$ & \\
\hline Age (years) & $69.0(51.0-79.0)$ & $65.0(49.0-78.0)$ & $77.0(69.3-85.0)$ & $<0.001$ & $68.0(51.0-79.0)$ & $75.5(69.0-82.3)$ & 0.031 \\
\hline Sex & & & & 0.684 & & & 0.620 \\
\hline Male & $376(50.5)$ & $318(50.2)$ & $58(52.3)$ & & $367(50.6)$ & $9(45.0)$ & \\
\hline Female & $369(49.5)$ & $316(49.8)$ & $53(47.7)$ & & $358(49.4)$ & $11(55.0)$ & \\
\hline \multicolumn{8}{|l|}{ Vital sign } \\
\hline Systolic blood pressure (mmHg) & $129.0(113.0-145.0)$ & $132.0(118.0-147.0)$ & $106.0(85.5-124.8)$ & $<0.001$ & $129.0(114.0-145.0)$ & $118.5(95.3-133.8)$ & 0.023 \\
\hline Diastolic blood pressure (mmHg) & $73.0(61.0-83.0)$ & $75.0(64.0-84.0)$ & $57.0(47.0-69.0)$ & $<0.001$ & $74.0(61.5-83.0)$ & $63.5(50.5-72.8)$ & 0.040 \\
\hline Heart rate (/min) & $89.0(74.0-105.0)$ & $87.0(73.0-102.0)$ & $103.5(83.0-117.0)$ & $<0.001$ & $88.0(74.0-105.0)$ & $95.5(78.8-112.0)$ & 0.237 \\
\hline Respiratory rate (/min) & $20.0(20.0-20.0)$ & $20.0(20.0-20.0)$ & $20.0(20.0-24.0)$ & $<0.001$ & $20.0(20.0-20.0)$ & $20.0(18.5-23.5)$ & 0.473 \\
\hline Body temperature $\left({ }^{\circ} \mathrm{C}\right)$ & $37.0(36.4-37.8)$ & $36.9(36.4-37.6)$ & $37.4(36.6-38.7)$ & 0.001 & $37.0(36.4-37.8)$ & $37.4(36.8-38.4)$ & 0.129 \\
\hline Mental status & & & & $<0.001$ & & & $<0.001$ \\
\hline Alert & $711(95.4)$ & $628(99.1)$ & $83(74.8)$ & & $699(96.4)$ & $12(60.0)$ & \\
\hline Verbal response & $24(3.2)$ & $5(0.8)$ & $19(17.1)$ & & $19(2.6)$ & $5(25.0)$ & \\
\hline Painful response & $10(1.3)$ & $1(0.2)$ & $9(8.1)$ & & $7(1.0)$ & $3(15.0)$ & \\
\hline Unresponsive & $0(0)$ & $0(0)$ & $0(0)$ & & $0(0)$ & $0(0)$ & \\
\hline \multicolumn{8}{|l|}{ Laboratory finding } \\
\hline White blood cell $\left(\times 10^{3} / \mu \mathrm{L}\right)$ & $9.7(7.2-13.3)$ & $9.6(7.2-13.0)$ & $10.6(7.7-18.5)$ & 0.004 & $9.7(7.2-13.1)$ & $14.3(7.9-23.5)$ & 0.010 \\
\hline Neutrophil $\left(\times 10^{3} / \mu \mathrm{L}\right)$ & $7.6(5.0-11.1)$ & $7.4(4.8-10.7)$ & $9.1(6.1-15.7)$ & $<0.001$ & $7.5(5.0-10.9)$ & $12.0(6.8-18.7)$ & 0.003 \\
\hline Lymphocyte $\left(\times 10^{3} / \mu \mathrm{L}\right)$ & $1.2(0.7-1.8)$ & $1.3(0.8-1.9)$ & $0.8(0.5-1.4)$ & $<0.001$ & $1.2(0.8-1.8)$ & $0.7(0.5-2.0)$ & 0.112 \\
\hline Platelet $\left(\times 10^{3} / \mu \mathrm{L}\right)$ & $217.0(167.8-275.0)$ & $224.0(176.5-284.5)$ & $159.5(105.8-220.8)$ & $<0.001$ & $217.0(169.0-274.3)$ & $207.0(104.5-293.5)$ & 0.469 \\
\hline Blood urea nitrogen $(\mathrm{mg} / \mathrm{dL})$ & $16.0(12.0-21.0)$ & $15.0(11.0-20.0)$ & $22.5(18.0-34.0)$ & $<0.001$ & $16.0(12.0-21.0)$ & $25.0(12.8-50.0)$ & 0.005 \\
\hline Creatinine (mg/dL) & $0.9(0.8-1.1)$ & $0.9(0.8-1.1)$ & $1.1(0.8-1.5)$ & $<0.001$ & $0.9(0.8-1.1)$ & $1.5(1.0-2.7)$ & $<0.001$ \\
\hline Total bilirubin $(\mathrm{mg} / \mathrm{dL})$ & $1.0(0.6-1.8)$ & $1.0(0.6-1.8)$ & $1.2(0.6-2.4)$ & 0.165 & $1.0(0.6-1.8)$ & $1.0(0.7-1.8)$ & 0.719 \\
\hline $\mathrm{C}$ - reactive protein $(\mathrm{mg} / \mathrm{dL})$ & $2.5(0.4-11.0)$ & $1.8(0.3-9.1)$ & $9.4(3.0-19.3)$ & $<0.001$ & $2.4(0.3-10.8)$ & $7.4(5.2-16.5)$ & 0.006 \\
\hline Blood culture, performed & $370(49.7)$ & $283(44.6)$ & $87(80.6)$ & $<0.001$ & $351(48.4)$ & $19(95.0)$ & $<0.001$ \\
\hline Result of blood culture & & & & $<0.001$ & & & 0.120 \\
\hline No growth & $273(74.1)$ & $233(82.3)$ & $41(47.1)$ & & $260(74.1)$ & $13(68.4)$ & \\
\hline Klebsiella pneumoniae & $41(11.1)$ & $25(8.8)$ & $16(18.4)$ & & $40(11.4)$ & $1(5.3)$ & \\
\hline Escherichia coli & $26(7.0)$ & $13(4.6)$ & $13(14.9)$ & & $24(6.8)$ & $2(10.5)$ & \\
\hline Enterococcus spp. & $11(3.0)$ & $4(1.4)$ & $7(8.0)$ & & $10(2.8)$ & $2(10.5)$ & \\
\hline Staphylococcus spp. & $4(1.1)$ & $2(0.7)$ & $2(2.3)$ & & $3(0.9)$ & $1(5.3)$ & \\
\hline Others & $14(3.8)$ & $6(2.1)$ & $8(9.2)$ & & $14(4.0)$ & $0(0.0)$ & \\
\hline
\end{tabular}

Quantitative data are expressed as median (interquartile range), categorical data are presented as number of subjects (percentages). Mann - Whitney U test was used for continuous variable analysis, while chi-squared test or Fisher's exact test were used for categorical variable analysis as appropriate.

ICU: intensive care unit. 

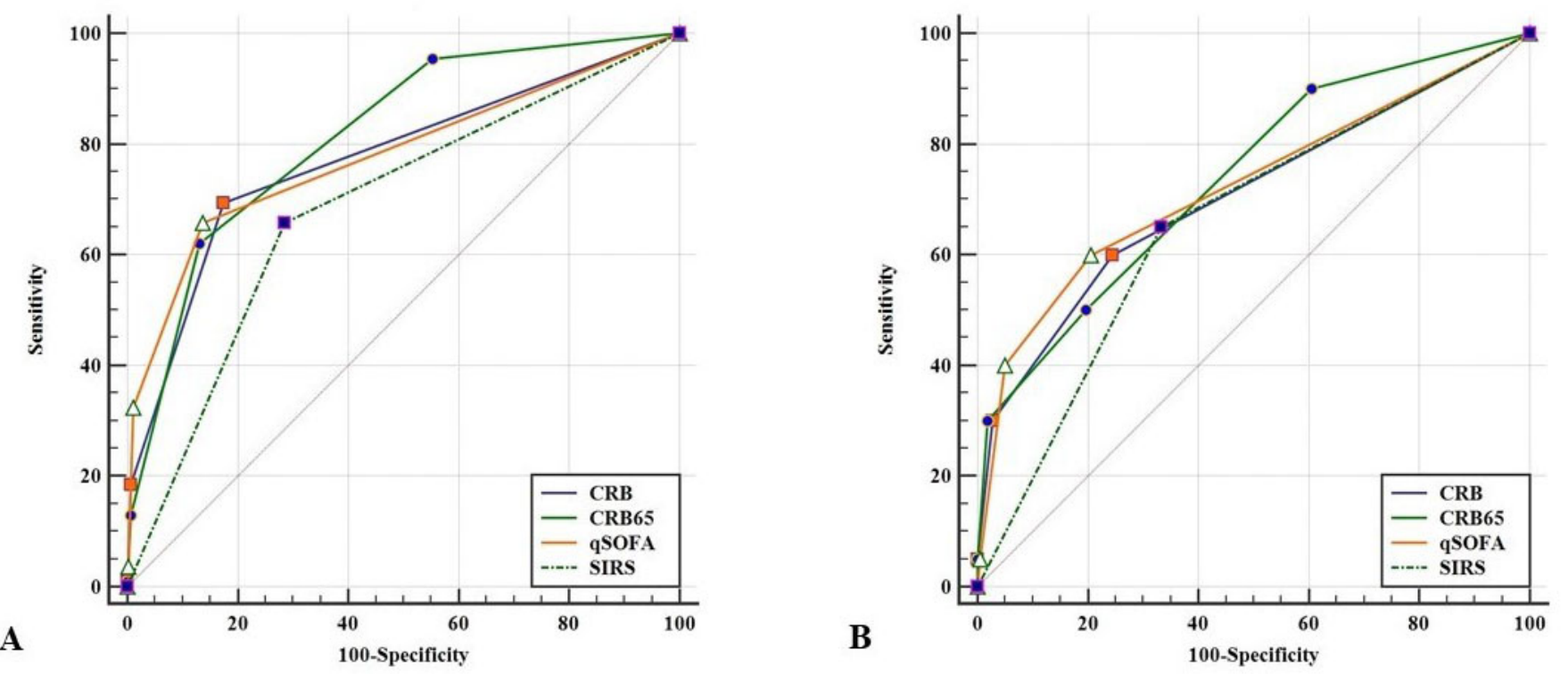

F I G U R E 1. The receiver operating characteristic curve for CRB, CRB-65, qSOFA, and SIRS for the ICU admission (A) and In-hospital mortality (B).

\section{Methods}

\subsection{Study Design, Setting, and Population}

This retrospective study conducted at an urban tertiary academic hospital with more than 65,000 ED patient visits annually. The medical data of patients who presented in the ED from February 2018 through March 2020 were collected from electronic medical records (EMRs) after screening for the study eligibility criteria. The inclusion criteria were (1) age 18 years or higher at the time of the ED visit; (2) diagnosis of acute cholecystitis or acute cholangitis, with or without a stone in the common bile duct (CBD) based on abdominal computed tomography (CT) findings. CT images were reviewed by a radiologist, but not by the same person. Patients with missing data for the initial vital signs, laboratory findings, and result of ED treatment were excluded.

\subsection{Data Collection and Outcome Measurement}

Data collected from the EMR included (1) patient demographics, including sex and age; (2) initial vital signs in the ED, including systolic blood pressure (SBP), diastolic blood pressure (DBP), pulse rate (PR), respiratory rate (RR), body temperature (BT), and mental status; (3) clinical details, including laboratory findings such as white blood cell (WBC) count, neutrophil count, lymphocyte count, platelet count, levels of Creactive protein (CRP), serum blood urea nitrogen $(\mathrm{BUN})$, and serum creatinine; (4) the result of ED treatment (i.e., discharge, admission to the general ward $[\mathrm{GW}]$, and intensive care unit [ICU] admission). The main outcome was ICU admission. The in-hospital mortality was also analyzed as a secondary outcome.

This study was approved by the Institutional Review Board of Ewha Womans University Mokdong Hospital (No. EUMC 2020-06-043).

\subsection{Statistical Analysis}

We conducted subgroup analyses for the groups that were stratified on the basis of the following criteria in line with the primary and secondary outcomes. The ICU admission and non-ICU admission groups included patients with ICU admission and discharge/GW admission, respectively. The non-survivor and survivor groups included patients who died in the hospital or survived until discharge, respectively.

The differences in the baseline characteristics were summarized using the Mann-Whitney $U$ test for continuous variables and the Pearson's chi-square test or Fisher's exact test for categorical variables, as appropriate. Continuous variables are presented as median with interquartile range whereas categorical variables are presented as count (percentage) and evaluated by using the area under the receiver operating characteristic (AUROC) curve. We compared the predictive accuracy of the CRB, CRB-65, q-SOFA, and SIRS for ICU admission and inhospital mortality by using calculations of AUROC and the $95 \%$ confidence intervals (CI). The optimal cutoff values of each scoring system were determined by the Youden Index of ROC curves [6]. Moreover, the sensitivity, specificity, positive and negative likelihood ratio (+LR and $-\mathrm{LR}$ ), and positive and negative predictive value (PPV and NPV) with $95 \% \mathrm{CI}$ were used to estimate the prognostic accuracy of each criteria for the proposed cutoff points. The significance level was considered as a $p$-value $<0.05$. Statistical analyses were performed using SPSS 22.0 (IBM Corp., Armonk, NY, USA). The ROC curve analysis was performed using the MedCalc Statistical Software version 19.4.1 (MedCalc Software Ltd, Ostend, Belgium; https://www.medcalc.org; 2020). The method of DeLong et al. [7] for the calculation of the standard error of the area under the curve (AUC) and of the difference between two AUCs were used.

\section{Results}


TA B L E 2. Score distribution of CRB, CRB - 65, qSOFA, and SIRS according to ICU admission and in - hospital mortality rates.

\begin{tabular}{|c|c|c|c|c|c|c|c|}
\hline \multirow[t]{2}{*}{ Variable } & \multirow[t]{2}{*}{ Total $(\mathrm{n}=745)$} & \multicolumn{2}{|c|}{ ICU admission } & \multirow[t]{2}{*}{ p-value } & \multicolumn{2}{|c|}{ In - hospital mortality } & \multirow[t]{2}{*}{ p-value } \\
\hline & & $\begin{array}{c}\text { Non-ICU admission } \\
\qquad(\mathrm{n}=634)\end{array}$ & $\begin{array}{l}\text { ICU admission } \\
\qquad(\mathrm{n}=111)\end{array}$ & & $\begin{array}{l}\text { Survivors } \\
(\mathrm{n}=725)\end{array}$ & $\begin{array}{l}\text { Non-survivors } \\
\quad(n=20)\end{array}$ & \\
\hline CRB & & & & $<0.001$ & & & $<0.001$ \\
\hline 0 & $556(74.6)$ & $523(82.5)$ & $33(29.7)$ & & $548(75.6)$ & $8(40.0)$ & \\
\hline 1 & $163(21.9)$ & $107(16.9)$ & $56(50.5)$ & & $157(21.7)$ & $6(30.0)$ & \\
\hline 2 & $25(3.4)$ & $4(0.6)$ & $21(18.9)$ & & $20(2.8)$ & $5(25.5)$ & \\
\hline 3 & $1(0.1)$ & $0(0)$ & $1(0.9)$ & & $0(0)$ & $1(5.0)$ & \\
\hline CRB-65 & & & & $<0.001$ & & & $<0.001$ \\
\hline 0 & $288(38.7)$ & $283(44.6)$ & $5(4.5)$ & & $286(39.4)$ & $2(10.0)$ & \\
\hline 1 & $304(40.8)$ & $268(42.3)$ & $36(32.4)$ & & $296(40.8)$ & $8(40.0)$ & \\
\hline 2 & $133(17.9)$ & $79(12.5)$ & $54(48.6)$ & & $129(17.8)$ & $4(20.0)$ & \\
\hline 3 & $19(2.6)$ & $4(0.6)$ & $15(13.5)$ & & $14(1.9)$ & $5(25.0)$ & \\
\hline 4 & $1(0.1)$ & $0(0.0)$ & $1(0.9)$ & & $0(0.0)$ & $1(5.0)$ & \\
\hline qSOFA & & & & $<0.001$ & & & $<0.001$ \\
\hline 0 & $584(78.4)$ & $547(86.3)$ & $37(33.3)$ & & $576(79.4)$ & $8(40.0)$ & \\
\hline 1 & $117(15.7)$ & $80(12.6)$ & $37(33.3)$ & & $113(15.6)$ & $4(20.0)$ & \\
\hline 2 & $39(5.2)$ & $6(0.9)$ & $33(29.7)$ & & $32(4.4)$ & $7(35.0)$ & \\
\hline 3 & $5(0.7)$ & $1(0.2)$ & $4(3.6)$ & & $4(0.6)$ & $1(5.0)$ & \\
\hline SIRS & & & & $<0.001$ & & & 0.011 \\
\hline 0 & $254(34.1)$ & $242(38.2)$ & $12(10.8)$ & & $253(34.9)$ & $1(5.0)$ & \\
\hline 1 & $237(31.8)$ & $211(33.3)$ & $26(23.4)$ & & $231(31.9)$ & $6(30.0)$ & \\
\hline 2 & $161(21.6)$ & $117(18.5)$ & $44(39.6)$ & & $154(21.2)$ & $7(35.0)$ & \\
\hline 3 & $86(11.5)$ & $60(9.5)$ & $26(23.4)$ & & $80(11.0)$ & $6(30.0)$ & \\
\hline 4 & $7(0.9)$ & $4(0.6)$ & $3(2.7)$ & & $7(1.0)$ & $0(0)$ & \\
\hline
\end{tabular}

The values are presented as number (\%). Chi - squared test or Fisher's exact test were used as appropriate.

CRB: Confusion, Respiratory rate, Blood pressure; CRB - 65: Confusion, Respiratory rate, Blood pressure, age > 65; qSOFA: quick Sequential Organ Failure Assessment; SIRS: Systemic Inflammatory Response Syndrome; ICU: intensive care unit.

\subsection{General Characteristics of Study Patients}

A total of 745 patients were included in this study. The baseline characteristics of the study participants are presented in Table 1 . The mean age of the patients in this study population was 65.1 years, and $50.5 \%$ were male. A total of $111(14.8 \%)$ patients were admitted to the ICU, and the in-hospital mortality was $20(2.7 \%)$. Regarding vital signs, the ICU admission group had significantly lower SBP, DBP, higher PR, RR, and BT than the-non-ICU admission group. On evaluation of the laboratory findings, the ICU admission group had significantly higher values of WBC, neutrophil, BUN, creatinine, and CRP and lower values of lymphocyte and platelet than the non-ICU admission group.

With regard to the in-hospital mortality, the non-survivor group had significantly lower SBP and DBP than the survivor group. The analysis of laboratory findings showed that the non-survivor group had significantly higher values of WBC, neutrophil, BUN, creatinine, and CRP than the survivor group.

\subsection{Score Distribution of CRB, CRB-65, qSOFA, and SIRS According to ICU Admission and In-hospital Mortality}

The numbers of patients stratified according to their CRB, CRB-65, qSOFA, and SIRS scores are shown in Table 2. A comparison of the score distribution according to the ICU admission status, CRB, CRB-65, qSOFA, and SIRS showed significant differences in score distribution. Thus, the CRB and qSOFA, with three points in total, and the CRB-65 and SIRS, with four points in total, showed similar score distribution patterns. In the ICU admission group, the distribution from 1 to 3 points of CRB and qSOFA was relatively high, and the distribution from 2 to 4 points of the CRB- 65 and SIRS was relatively high. In the comparison of the score distribution according to the in-hospital mortality, the CRB, CRB65, qSOFA, and SIRS score distributions were significantly different, and the results were the same as that for the analysis based on ICU admission. 
TA B L E 3. Validation of CRB, CRB - 65, qSOFA, and SIRS for ICU admission and in - hospital mortality.

ICU admission

\begin{tabular}{|c|c|c|c|c|c|c|c|c|c|c|c|c|c|c|}
\hline \\
\hline CRB & 1 & 0.774 & $0.742-0.803$ & 69.44 & $59.8-77.9$ & 82.49 & $79.3-85.4$ & $3.973 .2-4.9$ & 0.37 & $0.3-0.5$ & 40.3 & $35.4-45.5$ & 94.1 & $92.2-95.5$ \\
\hline CRB - 65 & 2 & 0.816 & $0.786-0.843$ & 62.04 & $52.2-71.2$ & 86.91 & $84.0-89.4$ & $4.743 .7-6.1$ & 0.44 & $0.3-0.6$ & 44.7 & $38.6-50.9$ & 93.1 & $91.3-94.5$ \\
\hline qSOFA & 1 & 0.779 & $0.747-0.808$ & 65.74 & $56.0-74.6$ & 86.28 & $83.4-88.9$ & $4.793 .8-6.1$ & 0.40 & $0.3-0.5$ & 44.9 & $39.1-50.9$ & 93.7 & $91.9-95.1$ \\
\hline SIRS & 2 & 0.686 & $0.651-0.719$ & 65.74 & $56.0-74.6$ & 71.45 & $67.8-74.9$ & $2.301 .9-2.8$ & 0.48 & $0.4-0.6$ & 28.2 & $24.6-32.0$ & 92.4 & $90.4-94.1$ \\
\hline \multicolumn{15}{|c|}{ In - hospital mortality } \\
\hline $\mathrm{CRB}$ & 1 & 0.707 & $0.673-0.739$ & 60.00 & $36.1-80.9$ & 75.59 & $72.3-78.7$ & $2.461 .7-3.6$ & 0.53 & $0.3-0.9$ & 6.3 & $4.4-9.0$ & 98.6 & $97.6-99.2$ \\
\hline CRB - 65 & 2 & 0.735 & $0.702-0.766$ & 50.00 & $27.2-72.8$ & 80.28 & $77.2-83.1$ & $2.531 .6-4.0$ & 0.62 & $0.4-1.0$ & 6.5 & $4.2-10.0$ & 98.3 & $97.4-98.9$ \\
\hline qSOFA & 1 & 0.724 & $0.690-0.755$ & 60.00 & $36.1-80.9$ & 79.45 & $76.3-82.3$ & $2.922 .0-4.3$ & 0.50 & $0.3-0.9$ & 7.5 & $5.2-10.6$ & 98.6 & $97.7-99.2$ \\
\hline
\end{tabular}

CRB: Confusion, Respiratory rate, Blood pressure; CRB - 65: Confusion, Respiratory rate, Blood pressure, age > 65; qSOFA: quick Sequential Organ Failure Assessment; SIRS: Systemic Inflammatory Response Syndrome; ICU: intensive care unit; AUROC: area under the receiver operating characteristic; CI: confidence interval; +LR: positive likelihood ratio; - LR: negative likelihood ratio; PPV: positive predictive value; NPV: negative predictive value.

TA B L E 4. Comparison of ROC curves between CRB, CRB - 65, qSOFA and SIRS for ICU admission and in - hospital mortality.

\begin{tabular}{|c|c|c|c|c|}
\hline & & \multicolumn{2}{|c|}{ Difference between areas $(95 \% \mathrm{CI})$} & \multirow[t]{2}{*}{ p-value } \\
\hline \multicolumn{4}{|c|}{ ICU admission } & \\
\hline \multirow[t]{3}{*}{ CRB } & CRB-65 & 0.042 & $(0.014-0.071)$ & 0.004 \\
\hline & qSOFA & 0.005 & $(-0.032-0.042)$ & 0.793 \\
\hline & SIRS & 0.088 & $(0.022-0.153)$ & 0.009 \\
\hline \multirow[t]{2}{*}{ CRB-65 } & qSOFA & 0.037 & $(-0.007-0.081)$ & 0.098 \\
\hline & SIRS & 0.130 & $(0.068-0.192)$ & $<0.001$ \\
\hline qSOFA & SIRS & 0.093 & $(0.029-0.156)$ & 0.004 \\
\hline \multicolumn{5}{|c|}{ In-hospital mortality } \\
\hline \multirow[t]{3}{*}{ CRB } & CRB-65 & 0.028 & $(-0.043-0.099)$ & 0.435 \\
\hline & qSOFA & 0.017 & $(-0.008-0.041)$ & 0.178 \\
\hline & SIRS & 0.048 & $(-0.078-0.174)$ & 0.455 \\
\hline \multirow[t]{2}{*}{ CRB-65 } & qSOFA & 0.012 & $(-0.065-0.088)$ & 0.769 \\
\hline & SIRS & 0.076 & $(-0.060-0.212)$ & 0.271 \\
\hline qSOFA & SIRS & 0.065 & $(0.058-0.187)$ & 0.299 \\
\hline
\end{tabular}

ROC: receiver operating characteristic; CRB: Confusion, Respiratory rate, Blood pressure; CRB - 65: Confusion, Respiratory rate, Blood pressure, age > 65; qSOFA: quick Sequential Organ Failure Assessment; SIRS: Systemic Inflammatory Response Syndrome; ICU: intensive care unit; CI: confidence interval. 


\subsection{Validation of CRB, CRB-65, qSOFA, and SIRS for ICU Admission and In-hospital Mortality}

The ROC curves of qSOFA, CRB, CRB-65, and SIRS are depicted in Fig. 1. The AUROC values for predicting ICU admission were as follows: CRB, 0.774 (95\% CI $0.742-$ 0.803); CRB-65, 0.816 (95\% CI 0.786 - 0.843), qSOFA, 0.779 (95\% CI $03747-0.808)$, and SIRS 0.686 (95\% CI 0.651 0.719). (Table 3 ) AUROC of CRB-65 was 0.8 or higher, and showed good performance as a predictor. [8] In comparisons between the two criteria, the CRB-65 vs CRB ( $p=0.004)$, CRB-65 vs SIRS ( $p<0.001)$, CRB vs SIRS $p=0.009$ ), and $q$ SOFA vs SIRS $(p=0.004)$ findings were significantly different (Table 4, Fig. 1A).

For the in-hospital mortality, the AUROCs of CRB, CRB65 , SIRS, and qSOFA were 0.707 (95\% CI $0.673-0.739)$, 0.735 (95\% CI 0.0.702-0.766), 0.724 (95\% CI 0.690-0.755), and 0.659 (95\% CI $0.623-0.693)$, respectively. (Table 3) A comparison of the two criteria for in-hospital mortality showed no significant differences between criteria (Table 4, Fig. 1B).

The cutoff values for the sensitivity and specificity of each criteria are shown in Table 3. A CRB-65 score $\geq 2$ had a sensitivity and specificity of $69.44 \%$ (95\% CI $52.2-71.2)$ and $82.49 \%(95 \%$ CI $79.37-85.4)$ for ICU admission and $60.0 \%(95 \%$ CI $36.1-80.9)$ and $75.59 \%$ (95\% CI $72.3-78.7)$ for in-hospital mortality, respectively. Similarly, for CRB $\geq$ 1 , the sensitivity and specificity were $69.44 \%$ (95\% CI $59.8-$ $77.9)$ and $82.49 \%$ (95\% CI 79.3 - 85.4) for ICU admissions and $60 \%(95 \%$ CI $36.1-80.9)$ and $75.59 \%$ (95\% CI $72.3-78.7)$ for in-hospital mortality, respectively. A qSOFA $\geq 1$ had a sensitivity and specificity of $65.74 \%(95 \%$ CI $56.0-74.6)$ and $82.28 \%$ (95\% CI 83.4 - 88.9) for ICU admissions and 60\% (95\% CI $36.1-80.9)$ and $79.45 \%$ (95\% CI $76.3-82.3)$ for in-hospital mortality, respectively.

\section{Discussion}

The early recognition of sepsis and its severity are important for appropriate treatment, improving outcomes, and reducing costs [4]. Therefore, many screening tools have been proposed to identify disease severity, including the SOFA, qSOFA, Acute Physiology and Chronic Health Evaluation, Multiple Organ Dysfunction Score (MODS), National Early Warning Score (NEWS), and CURB-65 (confusion, urea $>7$ $\mathrm{mmol} / \mathrm{L}$, respiratory rate $\geq 30 /$ minute, low systolic $[<90$ $\mathrm{mmHg}$ ] or diastolic $[\leq 60 \mathrm{mmHg}]$ blood pressure, and age $\geq 65$ years) $[9-11]$.

In this study, the qSOFA, CRB, and CRB-65 were used for severity scoring in BTI patients. Both SOFA and qSOFA are representative screening tools for determining disease severity. The European Society of Intensive Care Medicine developed the SOFA in 1994 to determine the extent of organ dysfunction/failure or to measure the likelihood of it mortality [12]. The SOFA consists of six items that can determine the functions of respiration, coagulation, liver, cardiovascular, central nervous, and renal systems. With the new definition of sepsis in 2016, the Third International Consensus Definitions for Sepsis and Septic Shock (Sepsis-3) task force proposed the
qSOFA as a simple clinical criterion that can be used at the bedside [4]. The items of qSOFA include altered mentation, $\mathrm{SBP} \leq 100 \mathrm{mmHg}$, and respiratory rate $\geq 22 / \mathrm{min}$. Since the qSOFA was proposed, a number of validation studies have been published, including a comparison with the existing SIRS criteria, and various results have been reported [13-17]. The qSOFA has been reported to be a useful tool for predicting the clinical outcome of patients with pneumonia $[18,19]$ and blunt trauma [20]. According to the findings in a study by Singer et al, the qSOFA was a useful indicator for predicting the mortality of adult ED patients with and without suspected infection (AUROC of qSOFA for mortality 0.76 95\% CI 0.73 - 0.78) [21]. In this study, the AUROC of the qSOFA for ICU admission and in-hospital mortality of BTI patients who were admitted to the ED was 0.779 and 0.724 , respectively. The entity of study groups were different, but showed similar results as that in the previous studies.

The other scoring systems used in this study are the CRB and CRB-65. The CURB-65 was developed for predicting the outcomes of patients with community-acquired pneumonia and is widely used in the clinical setting $[22,23]$. The CURB65 has been verified in many studies and may be applied to diseases other than community-acquired pneumonia [2426]. The CRB-65, which excludes the urea level from the CURB-65, has the advantage of being able to determine the disease severity of a patient without any laboratory findings. In previous studies, the CRB and CRB-65 showed similar predictive ability to that of CURB-65 [27, 28]. Most of CRB-65 studies were conducted on pneumonia, sepsis, and infection-related diseases. According to a study by Kordo et al, the AUROC of CRB-65 for 28-day mortality of patients with suspected infection presenting to the ED was 0.77 (95\% CI 0.72-0.81)[29]. In this study, the AUROC of CRB-65 for ICU admission and in-hospital mortality of BTI patients was 0.816 and 0.735 , and was the highest among the qSOFA, CRB, CRB65 , and SIRS. In particular, the AUROC for ICU admission showed good performance at 0.816 .

In case of CRB-65 and qSOFA, there are common factors, such as consciousness, respiration, and blood pressure, and there are many studies that have compared the two [5, 30-33]. A comparison of qSOFA and CRB-65 performance showed various results for each study. Although there may be differences in the AUROC, both are commonly reported as tools that can be used to measure clinical prognosis. In this study, the qSOFA, CRB, and CRB-65 were found to be scoring tools with "adequate" performance, with an AUROC of 0.7 or higher. However, only the AUROC of CRB-65 for ICU admission showed the highest value above 0.8 , which was also statistically significant when compared to the AUROC of CRB.

In this study, we attempted to verify the severity screening tools that can be easily applied at the bedside without laboratory findings in BTI patients in the ED. Of the many proposed screening tools, the CRB, CRB-65, and qSOFA were selected, and a comparative analysis was conducted. The CRB-65 showed the highest AUROC for predicting ICU admission $(0.816$ [95\% CI $0.786-0.843])$ and in-hospital mortality (0.735 [95\% CI 0.0.702 - 0.766]), whereas the CRB and qSOFA showed acceptable predictive ability with an AUROC 
$>0.7$. The SIRS showed poor performance, with AUROCs of 0.686 and 0.659 for ICU admission and mortality, respectively.

This study had several limitations. First, this was a singlecenter retrospective study. Thus, a selection bias may be existed due to the limited sample size and study conduct at a single institute. Caution should be exercised in generalizing the results of this study, and further studies with multicenter, prospective design are required. Second, patients with BTI, especially those who were older adults, tended to have comorbidities, which may have resulted in multiorgan dysfunction syndrome and affected the prognosis. Third, as a large tertiary academic hospital that was a referral center, the institution received patients who were in poor condition and transferred from smaller hospitals and primary healthcare institutions.

\section{Conclusions}

The CRB-65 had good predictive performance for the initial assessment of patients with BTI and can be used as a useful screening tool to predict ICU admission in patients with BTI on presentation to the ED.

\section{ACKNOWLEDGMENTS}

We would like to acknowledge our ED staffs for their support.

\section{CONFLICTS OF INTEREST}

The authors declare that there is no conflict of interest regarding the publication of this article.

\section{DATA AVAILABILITY}

The data that support the findings of this study are available from the corresponding author upon reasonable request.

\section{FUNDING INFORMATION}

This work was supported by the National Research Foundation of Korea (NRF) grant funded by the Ministry of Education (No. 2020R1I1A1A01070555). The funders had no role in study design, data collection and analysis, decision to publish, or preparation of the manuscript.

\section{AUTHORS' CONTRIBUTIONS}

Conception and design: Y. H. Choi, J. H. Lee; Acquisition, analysis, and interpretation of data: H. Yeo, S. J. Bae, J. H. Lee; Drafting the manuscript for intellectual content: H. Yeo, S. J. Bae, J. H. Lee, K Kim; Statistical analysis: S. J. Bae, J. H. Lee. All authors reviewed, revised, and approved the manuscript for submissions. Study supervision: Y. H. Choi.

\section{REFERENCES}

[1] Shah T, Sterk E, Rech MA. Emergency department sepsis screening tool decreases time to antibiotics in patients with sepsis. Am J Emerg Med. 2018;36:1745-1748.
[2] Melzer M, Toner R, Lacey S, et al. Biliary tract infection and bacteraemia: presentation, structural abnormalities, causative organisms and clinical outcomes. Postgrad Med J. 2007;83:773-776.

[3] Lee C-C, Chang I-J, Lai Y-C, et al. Epidemiology and prognostic determinants of patients with bacteremic cholecystitis or cholangitis. American Journal of Gastroenterology. 2007;102:563-569.

[4] Singer M, Deutschman CS, Seymour CW, et al. The Third International Consensus Definitions for Sepsis and Septic Shock (Sepsis-3). Jama. 2016;315:801-810.

[5] Kolditz M, Scherag A, Rohde G, et al. Comparison of the qSOFA and CRB-65 for risk prediction in patients with community-acquired pneumonia. Intensive Care Med. 2016;42:2108-2110.

[6] Perkins NJ, Schisterman EF. The inconsistency of "optimal" cutpoints obtained using two criteria based on the receiver operating characteristic curve. American journal of epidemiology. 2006;163:670-675.

[7] DeLong ER, DeLong DM, Clarke-Pearson DL. Comparing the areas under two or more correlated receiver operating characteristic curves: a nonparametric approach. Biometrics. 1988;44:837-845.

[8] Hajian-Tilaki K. Receiver operating characteristic (ROC) curve analysis for medical diagnostic test evaluation. Caspian journal of internal medicine. 2013;4:627.

[9] Sadaka F, EthmaneAbouElMaali C, Cytron MA, et al. Predicting Mortality of Patients With Sepsis: A Comparison of APACHE II and APACHE III Scoring Systems. J Clin Med Res. 2017;9:907-910.

[10] Lind ML, Phipps AI, Mooney S, et al. Predictive value of three clinical criteria for sepsis (qSOFA, SIRS, and NEWS) with respect to shortterm mortality in allogeneic hematopoietic cell transplant recipients with suspected infections. Clin Infect Dis. 2020.

[11] Peres Bota D, Melot C, Lopes Ferreira F, et al. The Multiple Organ Dysfunction Score (MODS) versus the Sequential Organ Failure Assessment (SOFA) score in outcome prediction. Intensive Care Med. 2002;28:1619-1624.

[12] Vincent J-L, Moreno R, Takala J, et al. The SOFA (Sepsis-related Organ Failure Assessment) score to describe organ dysfunction/failure. Springer-Verlag; 1996.

[13] Vincent J-L, Martin GS, Levy MM. qSOFA does not replace SIRS in the definition of sepsis. Critical care. 2016;20:1-3.

[14] Raith EP, Udy AA, Bailey M, et al. Prognostic accuracy of the SOFA score, SIRS criteria, and qSOFA score for in-hospital mortality among adults with suspected infection admitted to the intensive care unit. Jama. 2017;317:290-300

[15] Haydar S, Spanier M, Weems P, et al. Comparison of QSOFA score and SIRS criteria as screening mechanisms for emergency department sepsis. Am J Emerg Med. 2017;35:1730-1733.

[16] Finkelsztein EJ, Jones DS, Ma KC, et al. Comparison of qSOFA and SIRS for predicting adverse outcomes of patients with suspicion of sepsis outside the intensive care unit. Critical care. 2017;21:73.

[17] Rudd KE, Seymour CW, Aluisio AR, et al. Association of the quick sequential (sepsis-related) organ failure assessment (qSOFA) score with excess hospital mortality in adults with suspected infection in low-and middle-income countries. Jama. 2018;319:2202-2211.

[18] Jiang J, Yang J, Jin Y, et al. Role of qSOFA in predicting mortality of pneumonia: a systematic review and meta-analysis. Medicine. 2018;97:e12634.

[19] Müller M, Guignard V, Schefold JC, et al. Utility of quick sepsis-related organ failure assessment (qSOFA) to predict outcome in patients with pneumonia. PLoS One. 2017;12:e0188913.

[20] Jawa RS, Vosswinkel JA, McCormack JE, et al. Risk assessment of the blunt trauma victim: the role of the quick Sequential Organ Failure Assessment Score (qSOFA). The American Journal of Surgery. 2017;214:397-401.

[21] Singer AJ, Ng J, Thode Jr HC, et al. Quick SOFA scores predict mortality in adult emergency department patients with and without suspected infection. Annals of emergency medicine. 2017;69:475-479.

[22] Brabrand M, Henriksen DP. CURB-65 Score is Equal to NEWS for Identifying Mortality Risk of Pneumonia Patients: An Observational Study. Lung. 2018;196:359-361.

[23] Liu JL, Xu F, Zhou H, et al. Expanded CURB-65: a new score system predicts severity of community-acquired pneumonia with superior efficiency. Sci Rep. 2016;6:22911. 
[24] Chalmers JD, Singanayagam A, Akram AR, et al. Severity assessment tools for predicting mortality in hospitalised patients with communityacquired pneumonia. Systematic review and meta-analysis. Thorax. 2010;65:878-883.

[25] Chalmers JD, Singanayagam A, Akram AR, et al. Safety and efficacy of CURB65-guided antibiotic therapy in community-acquired pneumonia. Journal of Antimicrobial Chemotherapy. 2011;66:416-423.

[26] Yamamoto S, Yamazaki S, Shimizu T, et al. Prognostic utility of serum CRP levels in combination with CURB-65 in patients with clinically suspected sepsis: a decision curve analysis. BMJ open. 2015;5:e007049.

[27] Man SY, Lee N, Ip M, et al. Prospective comparison of three predictive rules for assessing severity of community-acquired pneumonia in Hong Kong. Thorax. 2007;62:348-353.

[28] Chen YX, Wang JY, Guo SB. Use of CRB-65 and quick Sepsisrelated Organ Failure Assessment to predict site of care and mortality in pneumonia patients in the emergency department: a retrospective study. Crit Care. 2016;20:167.

[29] Saeed K, Wilson DC, Bloos F, et al. The early identification of disease progression in patients with suspected infection presenting to the emergency department: a multi-centre derivation and validation study. Crit Care. 2019;23:40.

[30] Chen Y-X, Wang J-Y, Guo S-B. Use of CRB-65 and quick Sepsis- related Organ Failure Assessment to predict site of care and mortality in pneumonia patients in the emergency department: a retrospective study. Critical care. 2016;20:167.

[31] Mark K, George N, Bozorgmehri S, et al. 30 qSOFA Outperforms CRB, CRB-65 and CRB-65 Plus: A Multicenter US Observational Study. Annals of emergency medicine. 2017;70:S13.

[32] $\mathrm{Su}$ Y, Tu G-w, Ju M-j, et al. Comparison of CRB-65 and quick sepsisrelated organ failure assessment for predicting the need for intensive respiratory or vasopressor support in patients with COVID-19. J Infect. 2020.

[33] George N, Elie-Turenne M-C, Seethala RR, et al. External Validation of the qSOFA Score in Emergency Department Patients With Pneumonia. The Journal of Emergency Medicine. 2019;57:755-764.

How to cite this article:Hansol Yeo, Sung Jin Bae, Yoon Hee Choi, Keon Kim, Jae Hee Lee. CRB-65: Predictor for Intensive Care Unit Admission in Patients with Biliary Tract Infection Presenting to An Emergency Department. Signa Vitae. 2020;16(2):134-141. doi:10.22514/sv.2020.16.0049. 